\title{
Évaluation de la performance des digues de protection contre les inondations
}

\section{SERRE} L. PEYRAS R. TOURMENT

Cemagref

Unité de recherche Ouvrages hydrauliques 3275, route Cézanne CS 4006 13182 Aix-en-Provence Cedex 5

damien.serre@cemagref.fr laurent.peyras@cemagref.fr remy.tourment@cemagref.fr

Y. DIAB

LGUEH

Laboratoire

de Génie urbain

Environnement et Habitat

Université

de Marne-la-Vallée

5. bd Descartes

Bát. Lavoisier

77454 Marne-la-Vallée

Cedex 2

ydiab@univ-mlv.fr

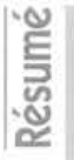

La France subit de fréquents épisodes de crues dévastatrices. Les inondations provoquent d'importants dégâts et les coûts induits sont considérables. Les digues de protection contre les inondations, souvent sousdimensionnées et mal entretenues, ont montré leurs faiblesses à plusieurs reprises et leurs ruptures augmentent la violence des inondations. A l'échelle nationale, le linéaire conséquent de digues (environ $7500 \mathrm{~km}$ ) et le manque de données sur l'ensemble de ce parc d'ouvrages compliquent leur gestion. A I'échelle du gestionnaire local se pose la question de l'optimisation des opérations de maintenance. Notre recherche vise à développer des méthodes d'évaluation de la performance des digues. Dans un premier temps, nous développons un modèle fonctionnel pour représenter les mécanismes de rupture des digues. Ce modèle permet de représenter des mécanismes sous forme de scénarios et de dégager les indicateurs pertinents de la performance des digues. A partir des informations disponibles (inspections visuelles détaillées, essais géophysiques ou géotechniques, données historiques...), nous agrégeons ces indicateurs par analyse multicritère appuyée sur la connaissance experte. Nous dégageons au final un indicateur global de performance des digues.

Mots-clés: digue, performance, analyse fonctionnelle, AMDE, analyse multicritère, indicateurs de performance, maintenance.

\section{Levee river performance assessment}

More and more floods occurred over the last decade in France and in the world, causing important damages and significant costs, Moreover, levees are often not well maintained, so they hardly resist to major floods and can break easily. These failures increase flood consequences. At French national scale, the length of levees, estimated to 7,500 kilometers, and the lack of data all along these infrastructures complicates their management.

At a local manager scale, the aim is to optimise maintenance operations. The goal of our research is to develop methods allowing the assessment of levees performance. Initially, we develop a functional model to represent levee failure mechanisms. This model allows to represent these mechanisms with scenarios and to build up the indicators used to assess levee performance. From data collected (detailed visual inspections, laboratory tests, historical data, etc.), we aggregate these indicators with a multicriteria analysis method based on expert knowledge. At the end we obtain a global levee performance indicator.

Key words: levee, performance, functional analysis, FMEA, multicriteria analysis, performance indicator, maintenance. 


\section{Introduction}

En France, mais aussi en Europe et, plus généralement, dans le monde, la fréquence des crues s'est accélérée lors de la dernière décennie et leur intensité a augmenté. Ces crues conduisent de plus en plus souvent à des ruptures de digues, ce qui a pour effet d'accroître les effets des inondations dans les zones censées être protégées par ces ouvrages. Citons quelques événements remarquables :

- les inondations dans le Sud de la France en 1999, 2002 et 2003;

- en Chine en 1998 le long du fleuve Yangze, où les ruptures de digues ont provoqué la mort de 2000 personnes, selon les chiffres officiels du gouvernement chinois :

- à Saint-Louis (États-Unis) le long du Mississippi en 1998, où des digues atteignant 15 mètres de hauteur se sont rompues ; très récemment (septembre 2005) à la Nouvelle-Orléans (États-Unis) suite au passage du cyclone Katrina. Le bilan provisoire à ce jour fait état de plusieurs milliers de morts et de plusieurs dizaines de milliards de dollars de dégâts.

En une génération, les problématiques liées aux digues ont profondément évolué, à l'instar d'autres domaines du génie civil. Les questions liées à la conception et à la réalisation des ouvrages se sont déplacées vers leur gestion et leur exploitation, avec l'objectif sous-jacent de la maîtrise des risques qu'ils induisent. Il s'agit donc de produire la meilleure gestion et la meilleure exploitation d'un parc de digues, et en particulier d'améliorer et d'optimiser les actions de maintenance, dans le souci permanent de maintenir sur le long terme un niveau de sécurité optimal pour l'aménagement hydraulique.

La maitrise des risques liés aux digues est fondamentale et les défaillances de ces ouvrages sont susceptibles d'avoir des conséquences en vies humaines ou économiques lourdes, comme l'ont montré les inondations récentes dans le Sud de notre pays : Languedoc-Roussillon en 1999 ( 36 victimes et $620 \mathrm{M} €$ de dommages), Gard en 2002 (23 victimes et $1200 \mathrm{M} €$ de dommages) et basse vallée du Rhône en 2003 ( 7 victimes et $1000 \mathrm{M} €$ de dommages).

En effet, la pression urbaine a abouti à autoriser la construction d'habitations dans le lit majeur des rivières, et les diques, souvent sous-dimensionnées et mal entretenues, ont montré leurs faiblesses à plusieurs reprises. La rupture de digues augmente la violence des inondations et peut accroittre les conséquences des inondations.

L'ancienneté des digues et l'absence de gestion rajsonnée de nombreux propriétaires ont provoqué une perte significative de l'information relative aux digues: la constitution de la digue, les travaux de confortement ultérieurs, etc. De plus, le linéaire important de digues en France $(7500 \mathrm{~km})$ complique leur gestion. Les gestionnaires et l'administration de contrôle sont conscients de l'importance de la mise en place de plans de gestion visant à mettre à niveau et à maintenir la sécurité des diques. Mais ce long linéaire pose une question majeure: par où commencer les actions de maintenance (Diab, 2002) ? Les gestionnaires ont un budget limité et il convient donc d'optimiser ce budget en planifiant les actions d'inspection et de maintenance.

Dans ce contexte, notre recherche vise à produire des méthodes pour évaluer la performance ${ }^{(1)}$ des digues de protection contre les inondations. L'objectif est de fournir aux gestionnaires des outils d'aide à la décision pour la planification des programmes d'inspection, de maintenance et de réparation. C'est l'objet du présent article. Les digues étant des ouvrages à grand linéaire, ces méthodes et outils visent à être intégrés in fine dans un système d'information géographique (SIG)

Dans une première partie introductive, nous présentons les digues de protection contre les inondations, leurs particularités et les différents mécanismes de rupture auxquels elles sont potentiellement soumises. Nous faisons également un exposé rapide de l'état du parc de digues en France.

Pour améliorer nos connaissances sur le fonctionnement et évaluer la performance des digues, nous développons une méthodologie permettant de modéliser les mécanismes de rupture. Pour cela, nous utilisons des méthodes de la sûreté de fonctionnement (Villemeur, 1988): l'analyse fonctionnelle (AF), l'analyse des modes de défaillances et de leurs effets (AMDE) et les arbres de défaillances. Ces méthodes appliquées aux digues vont permettre de modéliser les mécanismes de rupture sous forme de scénarios. Nous présentons ce travail dans la deuxième partie de l'article.

Nous cherchons ensuite à évaluer la performance des digues vis-à-vis de chaque scénario de rupture. Les scénarios vont nous indiquer quelles informations doivent être retenues pour construire les indicateurs de performance. Les méthodes multicritères d'aide à la décision, appuyées par l'expertise, vont nous permettre d'établir des règles pour évaluer les indicateurs de performance et pour les agréger. L'objectif est d'aboutir à l'évaluation de la performance globale d'une digue, en tenant compte de l'ensemble des mécanismes susceptibles de l'affecter. C'est l'objet de la troisième partie de l'article.

Il convient d'indiquer que ce travail s'inscrit dans un axe de recherche du Cemagref (organisme à vocation de contrôle et de suivi de barrages et de digues) visant à développer des bases de données sur les ruptures des ouvrages hydrauliques. I] a été réalisé dans le cadre d'une thèse en Sciences de l'information géographique, sous la direction de l'université Marne-laVallée (France). De fait, même si cette recherche présente une vocation applicative affirmée, la rigueur scientifique de notre démarche a été un objectif principal, en particulier dans l'application des méthodes de la sûreté de fonctionnement et de l'aide multicritère à la décision, et dans le recueil et la formalisation de la connaissance experte.

\footnotetext{
in Performance : aptitude de l'ouvrage à remplir les fonctions pour lesquelles il a été conçu (résister aux crues, ne pas se rompre, protéger les zones situẻes en arrière de la dígue).
} 


\section{Présentation des digues de protection contre les inondations} 1.1

\section{Définition et fonctionnement hydraulique des digues de protection contre les inondations}

Les digues de protection contre les inondations sont des ouvrages dont au moins une partie est construite en élévation au-dessus du niveau du terrain naturel et destinés à contenir épisodiquement un flux d'eau afin de protéger des zones naturellement inondables (Mériaux et al., 2001). Ces digues ne sont en charge qu'épisodiquement durant les crues des cours d'eau.

Les digues de protection contre les inondations font partie d'un système plus global : la vallée endiguée. On les trouve essentiellement le long des cours d'eau, parfois positionnées directement en contact avec la berge, ou éloignées de plusieurs mètres, voire de quelques centaines de mètres de celle-ci (Fig. 1).

La fonction principale des digues est la protection contre les inondations des zones qu'elles sont censées protéger. On peut décrire le fonctionnement hydraulique d'une vallée endiguée de la façon suivante:

- lors d'une crue, la rivière déborde de son lit mineur et inonde progressivement le lit majeur endigué ;

- l'endiguement limite l'emprise de l'inondation pour les crues faibles et moyennes, mais il surélève la ligne d'eau là où la présence des digues conduit à rétrécir sensiblement la largeur du lit naturel (c'est très souvent le cas dans la traversée des villes);

- l'écrêtement (phénomène qui amortit les débits maximaux par inondation d'espaces en lit majeur) est, de ce fait, limité lors des crues courantes; les zones protégées par des digues peuvent, dans certains cas, être inondées par le remous du fleuve à sa jonction avec un affluent, ou par les ruissellements issus de bassins versants latéraux dont les exutoires au fleuve se trouvent saturés, ou encore par remontée de la nappe phréatique;

- on aménage parfois des déversoirs permettant de protéger la digue contre le mécanisme de surverse, d'écrêter la crue en inondant une zone à enjeux limités; - pour les crues extrêmes, l'ensemble de la vallée est inondé soit à la suite du fonctionnement des déversoirs, soit par des ruptures de digues ; le fleuve ou la rivière recouvre ainsi l'ensemble de leur lit majeur, comme en l'absence de protections.

\section{2}

\section{Des ouvrages anciens et fragiles}

Les digues de protection contre les inondations sont pour la plupart des ouvrages anciens (les premières constructions remontant au Moyen Age). Il s'agit généralement d'ouvrages en remblai de terre, allant du limon au sable, parfois même au gravier. L'histoire de leur construction explique leur constitution. Les digues anciennes sont souvent des ouvrages construits par étapes à plusieurs périodes, en fonction de l'évolution des usages du fleuve ou des besoins de protection (Fig. 2).

Les digues anciennes ont été le plus souvent édifiées à partir de matériaux pris sur place. Ainsi, la variabilité des matériaux rentrant dans la constitution des ouvrages peut être grande, y compris le long d'un même cours d'eau (matériau sableux le long du bassin moyen et matériau limoneux en approchant de l'embouchure). Mais dans une section donnée, il s'agit généralement de remblais homogènes sans zonage et sans dispositifs particuliers de drainage interne.

L'absence de moyens lourds de terrassement et de compactage lors de l'édification des digues anciennes conduit à des remblais de relativement faible compacité sans ancrage particulier dans la fondation, laquelle n'a pas fait l'objet de traitement particulier pour assurer son étanchéité. Cette description rapide des digues anciennes de protection contre les inondations montre que ces ouvrages sont relativement fragiles.

A contrario, les digues les plus récentes (Fig. 3) font appel à des conceptions se rapprochant de celles des barrages. On y retrouve un zonage des matériaux avec séparation des fonctions d'étanchéité et de drainage.
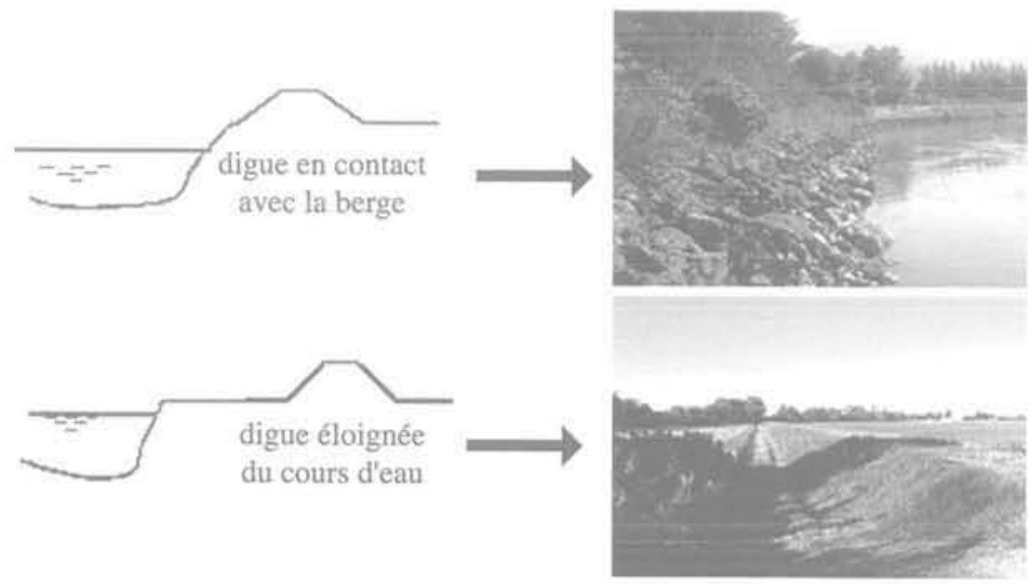

FIG.1 Les digues de protection contre les inondations dans leur environnement hydraulique.

River levees in their hydraulic environment. 


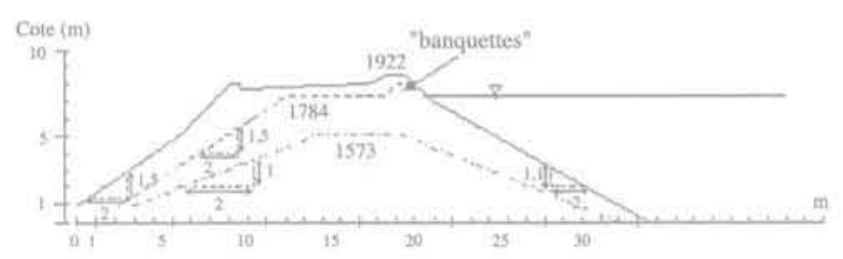

FIG. 2 Profil type d'une digue ancienne. Typical profile of an old levee.

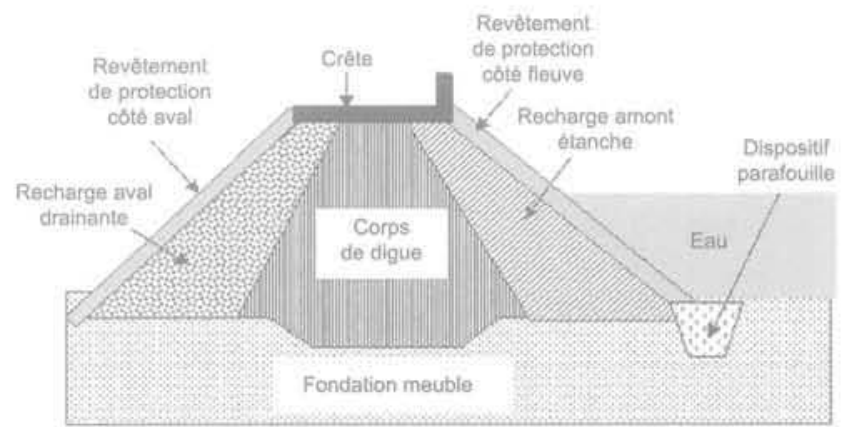

FIG.3 Profil type d'une digue récente. Typical profile of an old levee.
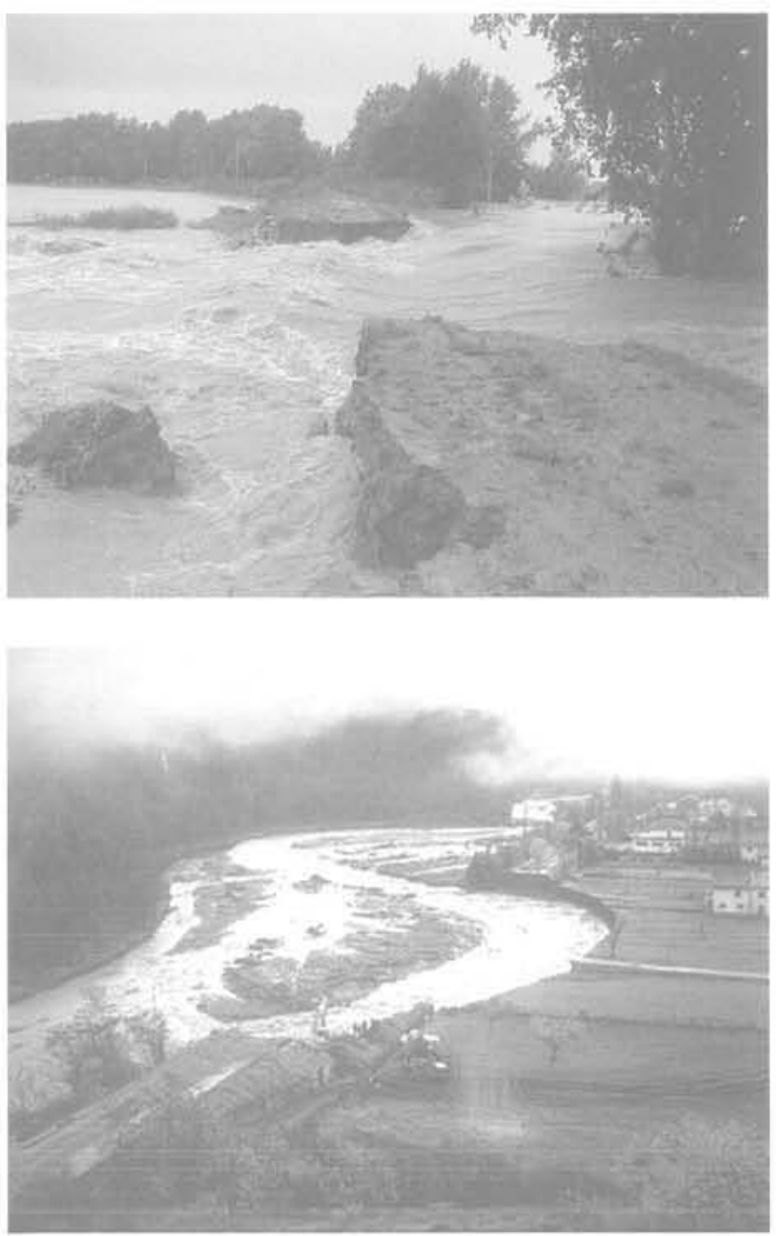

Le parc de digues de protection contre les inondations est constitué à $90 \%$ d'ouvrages en remblai (Mériaux et al., 2001). Ces digues sont donc susceptibles de subir des mécanismes de rupture propres à ce type d'ouvrage (Serre, 2005) : surverse, érosion interne, affouillement, glissement (Fig. 4).

Parmi ces mécanismes, la surverse et l'érosion interne sont à l'origine de la plupart des ruptures de digue:

- la surverse est à l'origine des principales ruptures de digue de protection contre les inondations. Ainsi, pour les digues de Loire, la surverse a été à l'origine de près de la moitié des ruptures lors des trois crues du milieu du XIX ${ }^{c}$ siècle (Mériaux et al., 2001). Dans plus des deux tiers des cas de surverse, on a pu identifier un point bas sur le profil en long de la digue qui a induit la concentration des débits. Des surélévations de la ligne d'eau sur la rive concave des courbes du fleuve, ou encore en amont de ponts ou seuils obstrués par des embâcles, peuvent également être à l'origine des surverses recensées;

- l'érosion interne est également à l'origine de nombreuses ruptures de digues (CFGB, 1997). En France, entre 1970 et 1997, seize manifestations d'érosion interne ont été répertoriées sur les digues de protection contre les inondations. Pour les crues du Rhône de 1993 et 1994, les mécanismes d'érosion interne sont à l'origine de la totalité des seize brèches constatées sur les digues de Camarque. Parmi celles-ci, treize sont dus à des terriers d'animaux et trois à des canalisations traversant la digue.
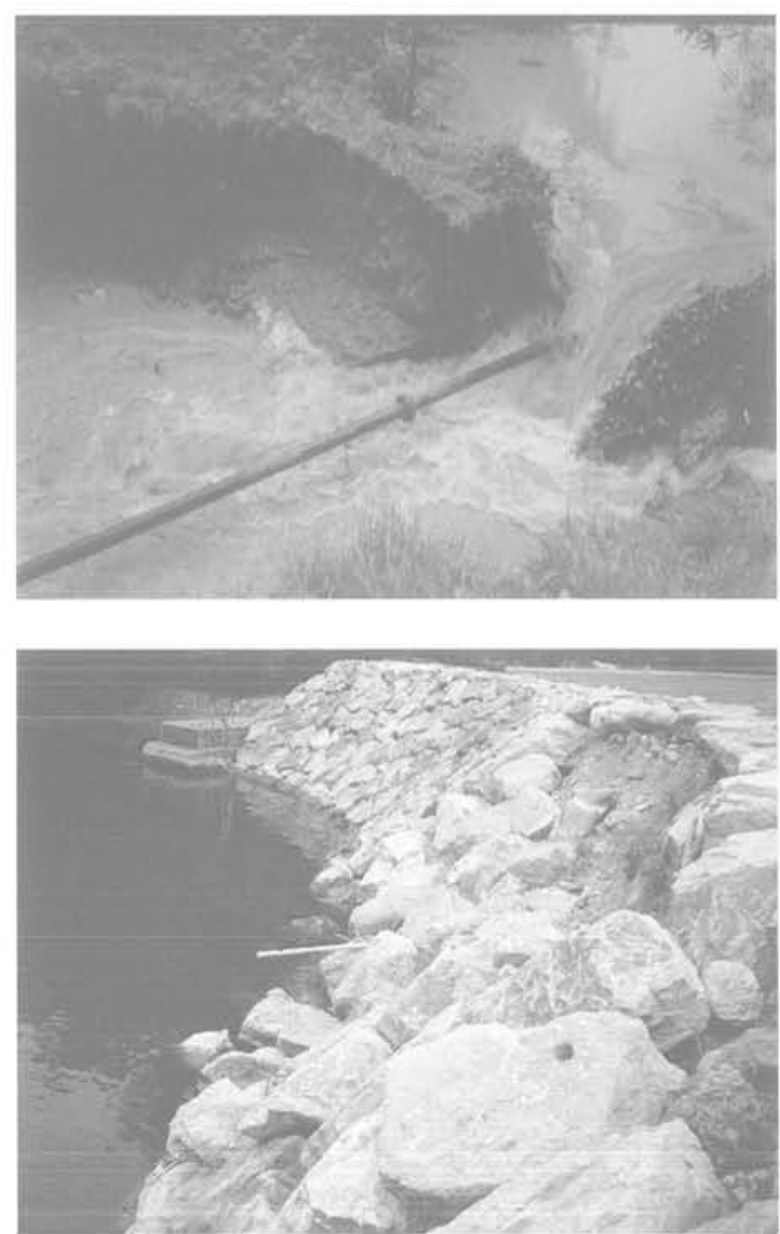

FIG.4 Les mécanismes de rupture des digues (surverse, érosion interne, affouillement, glissement). Illustration of levee failure mechanisms (overtopping, internal erosion, scour, slide). 


\section{Des enjeux importants et des dispositifs de gestion à améliorer}

Les zones protégées par les digues constituent des enjeux importants. La plupart des digues de protection contre les inondations intéressent la sécurité publique, et on estime le linéaire du parc de digues de protection contre les inondations à $7500 \mathrm{~km}$ sur le territoire métropolitain, protégeant une population estimée à 2 millions d'habitants et réparties sur une surface de quelque $15000 \mathrm{~km}^{2}$ (Mériaux et al., 2004).

Malgré ces enjeux, les digues de protection contre les inondations connaissent lors des crues majeures des défaillances pouvant conduire à des ruptures. Elles sont dues à un parc de digues globalement en mauvais état, mais aussi à une gestion des ouvrages inadaptée, voire inexistante.

En effet, les acteurs intervenant dans la gestion des digues sont très nombreux et trop souvent les propriétaires sont inconnus ou inactifs. L'absence dans de nombreux cas de maîtres d'ouvrages efficaces rend la gestion des digues perfectible.

Par ailleurs, les digues apparaissent mal ou non documentées et l'acquisition de données sur l'ensemble du linéaire est complexe et coûteuse du fait de l'hétérogénéité des digues.

Enfin, la gestion d'un parc d'ouvrage à grand linéaire, globalement méconnu, en mauvais état et présentant de fortes hétérogénéités, est complexe. Il est donc urgent de déployer un dispositif de gestion efficace pour améliorer la sécurité des digues. Aujourd'hui existent des méthodologies pour évaluer précisément l'état des digues, sur un troncon donné et sur une faible longueur (Fauchard et Mériaux, 2004). Toutefois, elles ne permettent pas d'évaluer la performance des digues sur l'ensemble du linéaire. Nous proposons donc des méthodes pour compléter le dispositif de gestion des digues (Serre, 2005).

2

\section{La modélisation des mécanismes de rupture des digues}

\section{1}

\section{Principe de la modélisation des mécanismes}

Nous cherchons ici à modéliser les mécanismes de rupture des digues sous forme de scénario, de façon à obtenir les informations nécessaires à l'évaluation de leur performance. Pour cela, il nous faut un modèle permettant de représenter tous les mécanismes de rupture et qui s'adapte à tous les types de digues.

Les modèles fonctionnels répondent au besoin de méthodes d'évaluation génériques. Nous avons exploité des outils destinés à modéliser les systèmes complexes et à représenter les liens logiques entre les successions de défaillance se produisant dans les ouvrages (les méthodes de la sûreté de fonctionnement). Le modèle fonctionnel de représentation des mécanismes est construit en trois étapes résumées à la figure 5 et développées dans la suite du texte.
(1) Analyse fonctionnelle $\rightarrow$ recherche des fonctions
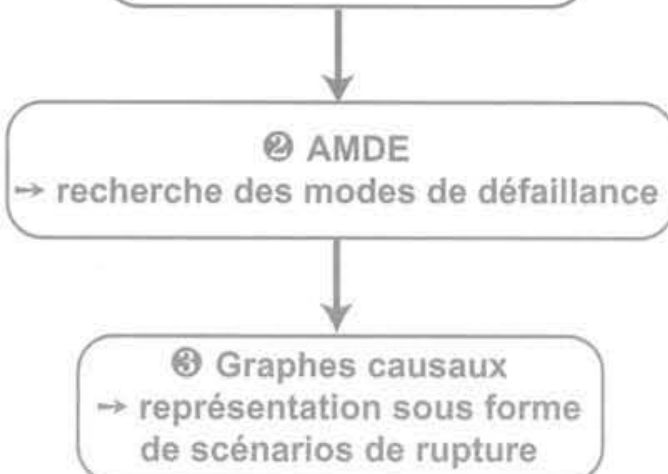

FG.5 Démarche de l'aide au diagnostic par expertise (Peyras, 2003).

Step of diagnostic aid with expertise.

\section{2}

\section{Analyse fonctionnelle des digues}

La première méthode à appliquer dans le cadre de la surete de fonctionnement est l'analyse fonctionnelle. Elle permet la compréhension et la description synthétique du fonctionnement d'un système. L'analyse fonctionnelle définit les limites du système considéré, son environnement et les fonctions qu'il remplit (Zwingelstein, 1996).

Pour réaliser l'analyse fonctionnelle d'une digue, il est nécessaire de fixer la précision de notre étude. Notre objectif étant une description fine des digues et de leurs mécanismes potentiels de rupture, nous réalisons l'analyse fonctionnelle à l'échelle spatiale du composant. Nous nous intéresserons donc aux fonctions accomplies par les composants des digues.

On procède ensuite à l'analyse structurelle des différentes catégories de digues. Nous analysons différents profils de digues, telles qu'elles sont observées en situation réelle. Cette analyse a conduit à proposer trois profils en travers types couvrant l'ensemble des digues rencontrées dans le parc français: les digues à étanchéité amont et les digues à étanchéité interne (Fig. 6), et, avec une représentation moindre, les digues en maçonnerie.

L'analyse structurelle des profils de digue permet de lister les différents composants constituant l'ouvrage, de déterminer la nature des matériaux les constituant, de repérer leur position géographique et de déterminer les interactions avec les autres composants. A titre d'illustration, nous développons l'analyse structurelle des digues à étanchéité amont (Tableau 1).

Les interactions des composants entre eux et avec les milieux extérieurs sont matérialisées au moyen de schémas (les blocs diagrammes fonctionnels) ètablis à l'échelle du composant (Fig. 7). Nous étudions les principales interactions des digues: celles liées aux contacts, aux sollicitations mécaniques et aux écoulements (flux hydrauliques et flux hydrodynamiques).

L'analyse des blocs diagrammes fonctionnels permet d'obtenir les fonctions de conception des composants, regroupées dans les tableaux d'analyse fonctionnelle (Tableau II) et classées selon l'origine de 

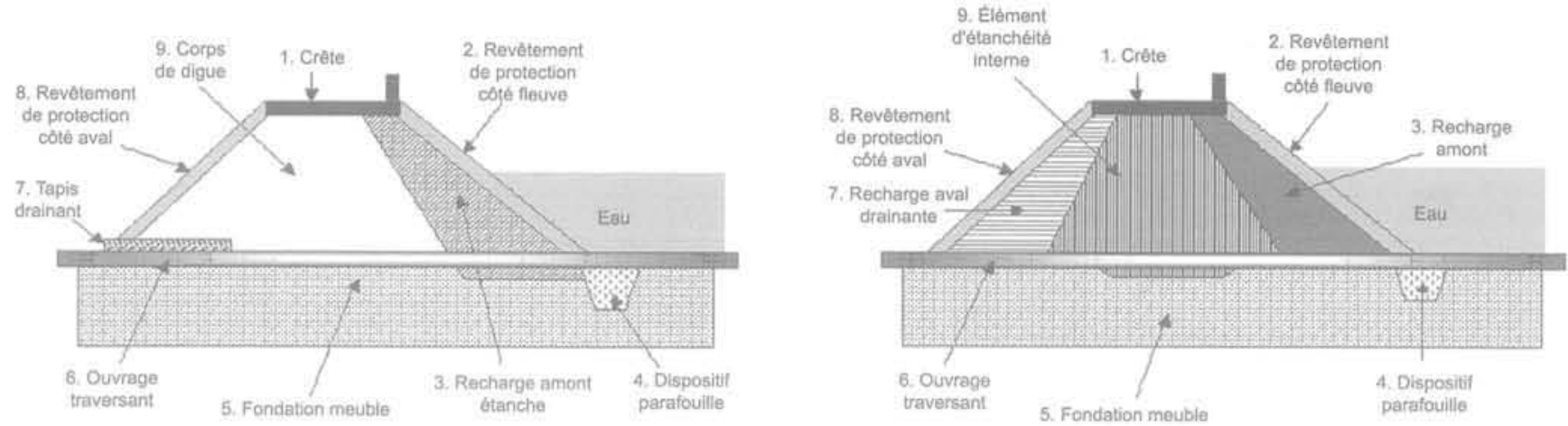

FG.6 Analyse structurelle des digues à étanchéité amont et des digues à étanchéité interne (Serre, 2005). Structural analysis of impervious upstream shoulder levee and levee with central core.

TABLEAU1 Les composants pour les digues en remblai à étanchéité amont (extrait). Component materials in levee with impervious upstream shoulder.

\begin{tabular}{|c|c|c|}
\hline Naméro & Composant & Nature possible \\
\hline 1 & Crểte & $\begin{array}{l}\text { Granulats } \\
\text { Terre }\end{array}$ \\
\hline 2 & Recharge amont étanche & $\begin{array}{l}\text { Terre végétale } \\
\text { Terre végétale ét grillage anti-fouisseur } \\
\text { Perré } \\
\text { Perré et terre végétale } \\
\text { Enrochements } \\
\text { Enrochements et terre végétale } \\
\text { Argiles compactées } \\
\text { Géomembrane }\end{array}$ \\
\hline
\end{tabular}

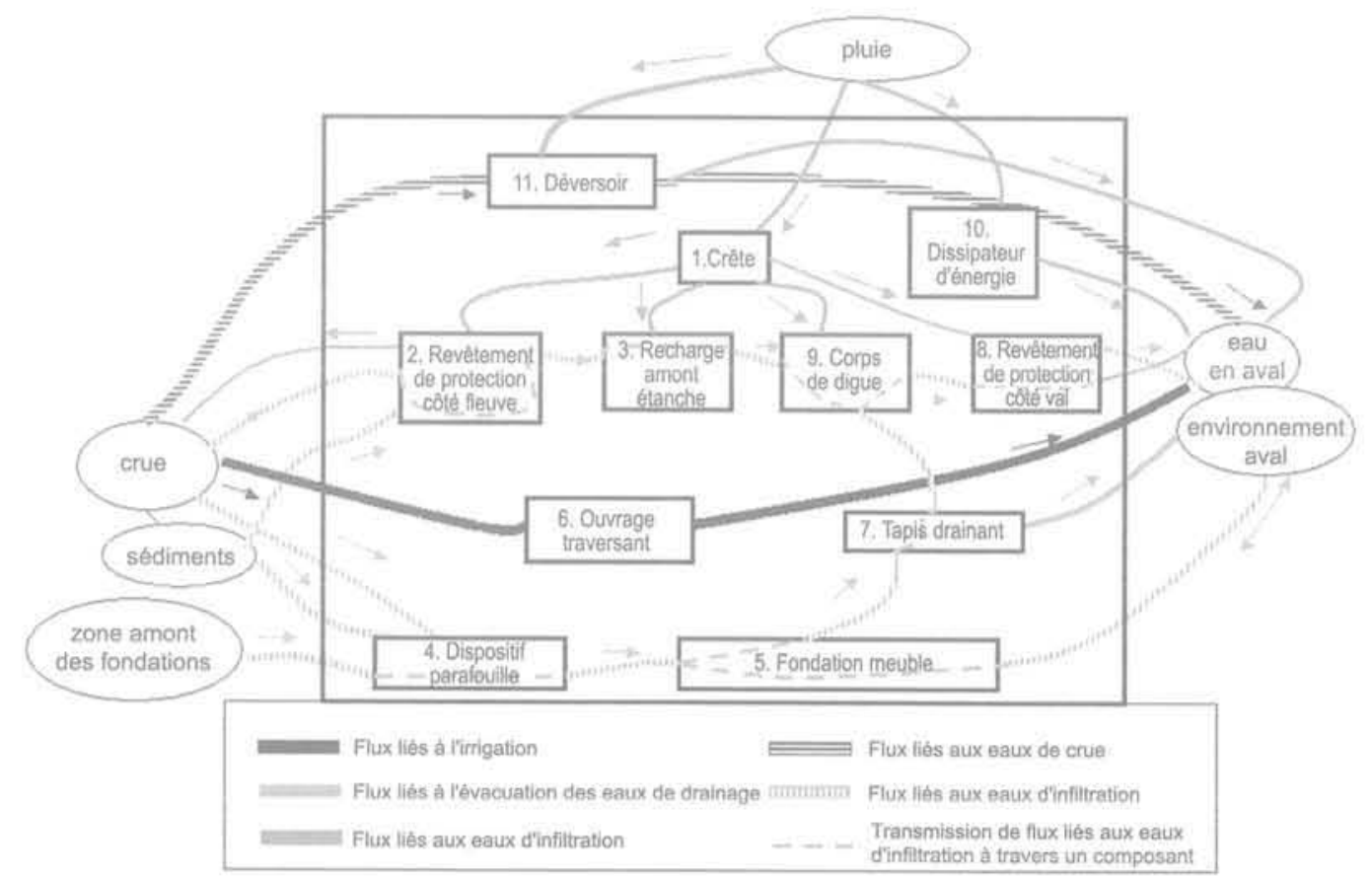

FIG.7 Bloc diagramme fonctionnel ( relations de flux hydrauliques $)$. Functional block diagram for a hydraulic flow relationship ». 
TABLEAUII Tableau d'analyse fonctionnelle (Serre 2005) (extrait). Functional analysis table.

\begin{tabular}{|c|c|c|c|}
\hline Numéro & Composant & Classification de la fonction & Fonctions de conception \\
\hline \multirow[t]{2}{*}{1} & Crête & Flux hydrauliques & $\begin{array}{l}\text { - limiter l'infiltration d'eau de pluie } \\
\text { - évacuer l'eau de ruissellement vers le } \\
\text { revêtement de protection côté val } \\
\text { - résister à l'érosion externe provenant des eaux dé } \\
\text { ruissellement } \\
\text { - résister ả l'ẻrosion externe au cours d'une crue }\end{array}$ \\
\hline & & Sollicitations mécaniques: & $\begin{array}{l}\text { - résister aux charges dues à la circulation } \\
\text { - résister aux développements des racines }\end{array}$ \\
\hline
\end{tabular}

l'interaction (contacts, sollicitations mécaniques, flux hydrauliques...). Au final, ces tableaux synthétisent l'analyse fonctionnelle des digues et récapitulent, pour chaque composant, les fonctions de conception accomplies par le composant.

\section{3}

\section{AMDE appliquée aux digues}

La méthode AMDE (analyse des modes de defaillance et de leurs effets) est apparue dans les années 60 dans le domaine de l'aéronautique et est actuellement une des méthodes de la sûreté de fonctionnement la plus utilisée dans l'industrie (spatiale, nucléaire, automobile...). L'AMDE est une méthode inductive d'analyse des défaillances potentielles d'un système. Elle considère, systématiquement, l'un après l'autre, chaque composant du système et analyse ses modes de défaillance, leurs causes et leurs effets.

Nous adaptons les techniques d'analyse de la méthode AMDE à notre contexte. Les résultats sont listés dans un tableau indiquant (Tableau III) :

- les composants (et leur numéro de nomenclature) correspondent à la décomposition structurelle;

- les fonctions de conception des composants obtenues à partir de l'analyse fonctionnelle;

- les modes de défaillance : la défaillance d'une fonction (fonction non réalisée) et la dégradation d'une fonction (fonction partiellement ou mal réalisée);
- les causes possibles des défaillances d'un composant, obtenues à partir des blocs diagrammes fonctionnels. Ces causes sont classées selon leur origine: les contacts avec les milieux extérieurs ou les composants environnants, les sollicitations mécaniques, les flux hydrauliques (ou hydrodynamique), l'état intrinsèque du composant (ses caractéristiques propres), les causes liées à la conception/réalisation du composant;

- les effets possibles d'un mode de défaillance, classés de façon analogue aux causes :

- les indicateurs (visuels, issus d'un instrument d'auscultation, les coefficients de sécurité) qui traduisent la manifestation des effets des défaillances. Ils sont détectés par différents moyens de détection.

En synthèse de l'AMDE appliquée aux digues de protection contre les inondations, nous disposons de leurs principaux modes de défaillance, de leurs causes, de leurs effets et de leurs indicateurs associés.

\section{Modèle fonctionnel pour les mécanismes de rupture des digues}

A ce stade, nous pouvons construire les scénarios de rupture des digues, en enchaînant les causes aux modes de défaillance, puis aux effets. Les scénarios sont ainsi modélisés par des successions de séquences de

TABLEAU III L'AMDE adaptée aux digues (Serre 2005) (extrait). Levee FMEA.

\begin{tabular}{|c|c|c|c|c|c|c|c|}
\hline $\mathrm{N}^{\circ}$ & Composant & $\begin{array}{l}\text { Fonction } \\
\text { de conception }\end{array}$ & $\begin{array}{l}\text { Mode } \\
\text { de défaillance }\end{array}$ & $\begin{array}{l}\text { Causes possibles } \\
\text { de la défaillance }\end{array}$ & $\begin{array}{l}\text { Effets possibles } \\
\text { de la défaillance }\end{array}$ & Indicateurs & $\begin{array}{l}\text { Moyens } \\
\text { de détection }\end{array}$ \\
\hline \multirow[t]{2}{*}{1} & Crête & $\begin{array}{l}\text { limiter } \\
\text { les infiltrations } \\
\text { d'eau de pluie }\end{array}$ & la fonction & $\begin{array}{l}\text { - Processus } \\
\text { conception- } \\
\text { réalisation: }\end{array}$ & - Flux hydrauliques: & & \\
\hline & & & $\begin{array}{l}\text { "limiter les } \\
\text { infiltrations } \\
\text { d'eau } \\
\text { de pluiex } \\
\text { est dégradée } \\
\text { ou est } \\
\text { défaillante }\end{array}$ & $\begin{array}{l}\text { - composition } \\
\text { de la crête } \\
\text { - Etat intrinsèque } \\
\text { du composant: } \\
\text { - fissuration } \\
\text { de la crète }\end{array}$ & $\begin{array}{l}\text { - eaux d'infiltration } \\
\text { dans le corps } \\
\text { de digue } \\
\text { - eaux d'infiltration } \\
\text { dans la recharge } \\
\text { amont étanche } \\
\text { - Sollicitations } \\
\text { mécaniques: } \\
\text { - sous-pressions } \\
\text { dans le corps } \\
\text { de dique }\end{array}$ & $\begin{array}{l}\text { - fuites sur } \\
\text { le parement aval }\end{array}$ & $\begin{array}{l}\text { - observation } \\
\text { visuelle } \\
\text { - piézomètres }\end{array}$ \\
\hline
\end{tabular}


défaillance fonctionnelle, représentant les processus physiques se produisant au sein du système et conduisant à des pertes ou dégradations de fonctions. Nous représentons les scénarios sous forme de graphes causaux : chaque mécanisme est modélisé dans un graphe causal décrivant les processus fonctionnels de dégradation et les enchaînements de variables correspondants (Peyras, 2003).

Le modèle de représentation des scénarios comporte trois catégories de variables: les variables "fonction» correspondant aux modes de défaillance, les variables "phénomène » regroupant les causes et effets des modes de défaillance et les variables «indicateur» correspondant aux manifestations des phénomènes.

Les variables " phénomène » sont renseignées par les variables « indicateur $»$, qui traduisent la manifestation des mécanismes (indicateur $\Rightarrow$ phénomène). Les combinaisons de variables "phénomène» vont conduire à la dégradation d'une (ou des) variable(s) "fonction», la perte de performance de cette dernière produisant à nouveau une nouvelle séquence de phénomènes (phénomènes $\Rightarrow$ fonction $\Rightarrow$ phénomènes). L'enchainement des défaillances sous forme "de phénomènes impliquant des dégradations de fonctions, impliquant de nouveaux phénomènes x constitue le modèle de représentation des mécanismes de rupture d'une digue sous forme de scénario (Fig, 8).

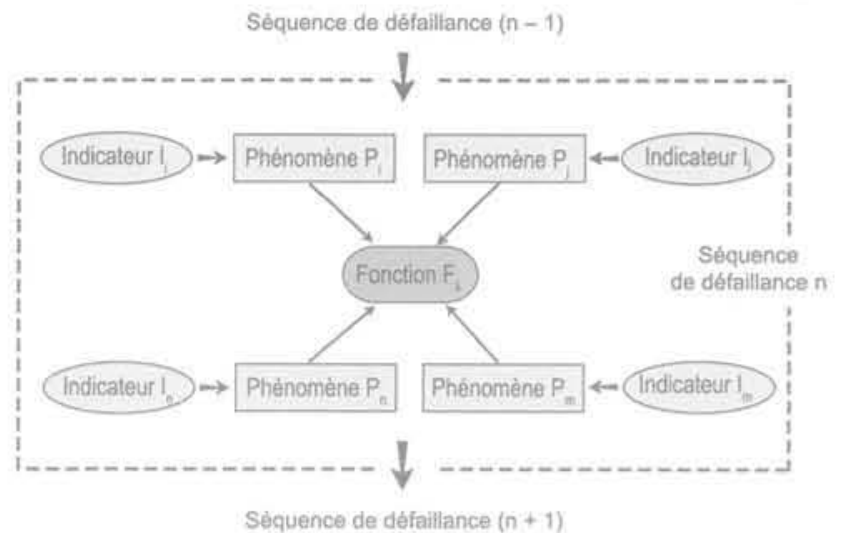

FG. 8 Modèle fonctionnel de représentation des mécanismes (Peyras, 2003).

Functional representation model of mechanisms.

Un tel modèle présente de nombreux avantages: il structure la connaissance experte sous forme de scénarios fonctionnels, il organise l'information liée aux mécanismes autour de trois catégories de variable (fonction, phénomène et indicateur) et il permet de prendre en compte les dégradations partielles et progressives des variables et les mécanismes non chronologiques.

Les phénomènes (les causes et effets des modes de défaillance) intervenant dans les mécanismes de rupture des digues sont répertoriés et classés selon l'origine:

- état intrinsèque du composant: déformation, fissuration, érosion, colmatage, composition..

- flux hydraulique : crue, eau d'infiltration, fuites, percolations, suintements..

- les sollicitations mécaniques: poussée hydrostatique, poids propre, sous pressions...
- les sollicitations hydromécaniques: érosion interne, érosion externe, colmatage...

- la conception et la réalisation: perméabilité, capacité de drainage...

Nous distinguons trois catégories d'indicateurs (les indicateurs traduisent les phénomènes dans les mécanismes)

- les indicateurs visuels sont détectés par l'observation visuelle du garde-digue ou de l'expert: érosion, végétation, ouvrages singuliers, terriers d'animaux, glissements..

- les indicateurs mesurés sont issus d'une mesure d'auscultation ou d'un essai in situ ou au laboratoire: piézométrie, débit, déformations, perméabilité, compacité...

- les indicateurs calculés sont obtenus à partir d'un calcul faisant intervenir des indicateurs auscultés et correspondent aux facteurs de stabilité intervenant dans les conditions d'états limites des digues : gradient hydraulique, charge hydraulique, coefficients de sécurité au glissement...

\section{5}

\section{Modélisation des mécanismes de rupture des digues: application à l'érosion interne}

Nous disposons d'un modèle fonctionnel pour représenter des scénarios et nous cherchons maintenant à renseigner une base de connaissances sur les mécanismes de rupture des digues. Le support de ce travail est la connaissance experte, contenue dans les documentations techniques relatives à la pathologie des digues: nous renvoyons le lecteur intéressé vers (Pilarczyk, 1998) et (Mériaux et al., 2001) qui sont les références principales sur le sujet. L'interview des experts est également utilisée en complément de l'analyse des recueils d'expertise.

A partir de la connaissance experte, nous déterminons les successions de défaillances fonctionnelles pour chaque mécanisme de rupture. Nous en déduisons les modes de défaillance en jeu, les phénomènes conduisant à ces modes de défaillances ou en résultants et les indicateurs permettant de détecter les phénomènes. Nous classons ces informations et nous procédons à des regroupements et à des choix terminologiques afin d'obtenir des informations structurées et homogènes, pouvant être intégrées ultérieurement dans des bases de données.

Nous pouvons alors construire les scénarios de rupture des digues correspondant à chaque mécanisme selon la représentation proposée dans notre modèle. Les mécanismes de rupture ainsi modélisés sous forme de scénario sont résumés ci-après pour le cas particulier des digues en remblai à étanchéité amont (Tableau IV).

A titre illustratif, nous appliquons le modèle fonctionnel proposé au mécanisme d'érosion interne d'une digue en remblai à étanchéité amont (scénario $n^{\circ} 4$ du tableau IV). Ce mécanisme comportent trois séquences de défaillance successives : i) la dégradation mécanique du revêtement de protection côté fleuve, conduisant à ii) la perte d'étanchéité de la recharge amont, ellemême conduisant à iii) des processus d'érosion interne dans le corps de digue.

Dans une première séquence de défaillance, plusieurs phénomènes sont susceptibles de dégrader la 
TABLEAU NN Les scénarios de rupture des digues à étanchéité amont.

Failure scenarios for levves with upstream impervious shoulder.

Numéro

Nom

Condition hydraulique

1

1
2
3
4
5
6

Surverse

Affouillement côté fleuve

Erosion interne en fondation

Érosion interne dans le corps de digue

Glissement cótẻ fleuve

Glissement côtẻ val

crue

crue ou régime normal de la rivière crue ou régime normal de la rivière

crue

décrue

crue performance de la fonction de protection du revêtement amont ffonction « le revêtement de protection côté fleuve résiste aux sollicitations mécaniques $x$, Fig. 9). Il s'agit des phénomènes: «végétation», "animaux fouisseurs , "chocs d'embâcles », " géo- morphologie de la rivière » et «composition du revêtement de protection côté fleuve m.

Lors de la deuxième séquence de défaillance, sous l'effets de phénomènes tels que les a racines dans la recharge amont étanche », « trous d'animaux fouisseurs

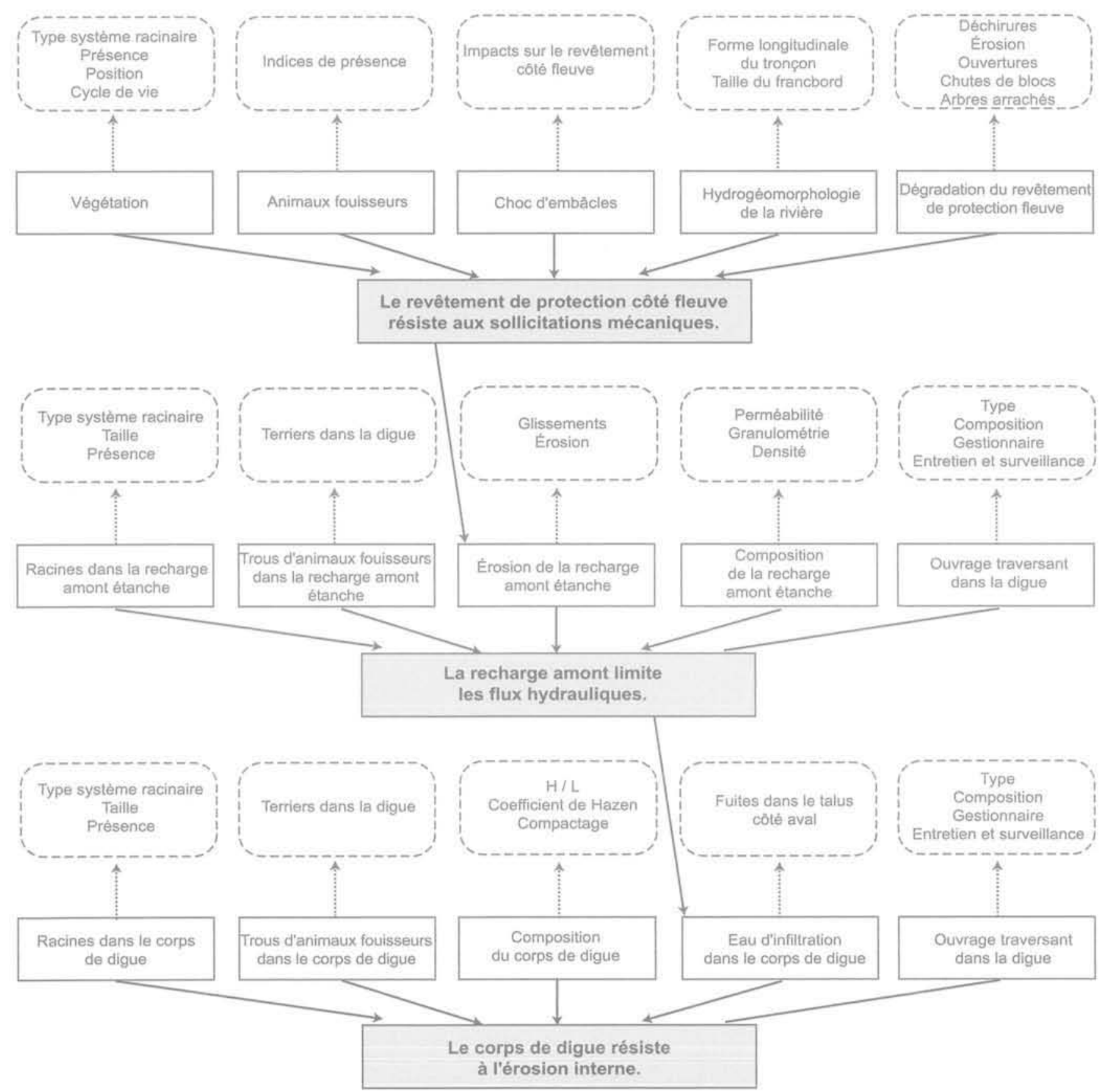

FIG.9 Scénario d'érosion interne dans les digues à étanchéité amont. Scenario for internal erosion in levee with impervious upstream shoulder. 
dans la recharge amont étanche », «érosion de la recharge amont étanche », "composition de la recharge amont étanche» et "ouvrages traversants dans la digue ), la fonction d'étanchéité de la recharge amont (fonction «la recharge amont étanche limite les flux hydrauliques m, Fig. 9) va se dégrader, conduisant à des infiltrations d'eau dans le corps de digue.

La troisième séquence de défaillance concerne le corps de digue. La fonction de résistance de la digue (fonction «le corps de digue résiste à l'érosion interne», Fig. 9) peut être affectée par les phénomènes « racines dans le corps de digue», "trous d'animaux fouisseurs dans le corps de digue », "composition du corps de digue », "eau d'infiltration dans le corps de digue » et "ouvrages traversants dans la digue ». Les effets de la perte de performance de cette fonction sont des phénomènes d'érosion interne dans le corps de digue, susceptibles de conduire à la rupture du tronçon.

Par l'analyse des processus physiques s'opérant dans les ouvrages et à chaque niveau de défaillance, nous avons recensé les fonctions de conception des composants qui vont se dégrader, les phénomènes conduisant à ces modes de défaillance ou en résultant et leurs indicateurs associés. Nous disposons donc de toutes les informations pour construire le scénario correspondant au mécanisme d'érosion interne des digues à étanchéité amont (Fig. 9).

\section{6}

\section{Synthèse de la modélisation des mécanismes de rupture des digues}

L'application de cette démarche aux diques a permis d'obtenir des résultats intéressants pour notre objectif d'évaluation de la performance des digues. L'analyse fonctionnelle fournit une modélisation cohérente des différents types de digues et établit une synthèse des fonctions, phénomènes et indicateurs intervenant dans les mécanismes de rupture. A partir de l'AMDE, nous proposons un modèle fonctionnel pour représenter les mécanismes sous forme de scénarios. Enfin, la connaissance experte permet de formaliser et recueillir toute l'information relative aux mécanismes et de représenter les mécanismes.

En termes applicatifs, cette démarche conduit à proposer trois types de profil de digue de protection contre les inondations, couvrant l'ensemble du parc français. L'analyse de la connaissance experte et l'interview des experts ont permis de recenser les informations relatives aux mécanismes de rupture: les indicateurs, les phéno-

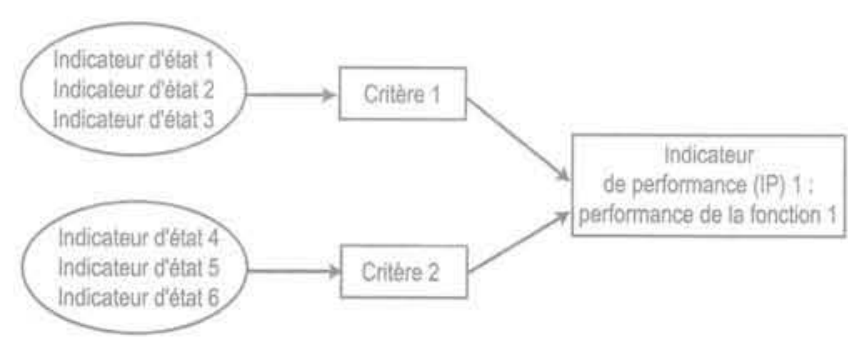

FIG.10 Hiérarchisation des indicateurs d'état, des critères et des indicateurs de la performance des digues.

Hierarchisation of condition indicators, criteria and performance indicators. mènes, les fonctions et leurs modes de défaillance. Ainsi, nous représentons sous la forme de scénarios fonctionnels l'ensemble des mécanismes de rupture des digues (6 scénarios pour les digues à étanchéité amont).

\section{3}

\section{Évaluation multicritère de la performance des digues}

\section{1}

\section{Principes de l'analyse multicritère pour l'évaluation de la performance des digues}

En génie civil, les méthodes multicritères sont souvent utilisées pour la gestion des ouvrages à grands linéaires: les réseaux d'eau potables (Le Gauffre et al. 2004), les réseaux d'assainissement (Diab, 2002). L'objectif de ces méthodes est l'évaluation des conditions de fonctionnement du système étudié. Notre problématique est analogue: à partir des scénarios de rupture des digues, nous souhaitons développer une méthode pour évaluer leur performance.

La démarche mise en œuvre repose sur l'utilisation d'outils d'analyse multicritère (Roy et Bouyssou, 1993). Cette démarche comporte différentes étapes. La performance d'une digue dépend de plusieurs critères, définis eux-mêmes à partir de combinaisons d'indicateurs d'état. II nous faut: i) déterminer les indicateurs d'état et les critères, ii) proposer des règles pour leur évaluation et iii) agréger les critères pour obtenir la valeur de la performance des digues vis-à-vis d'un mécanisme de rupture (Fig. 10).

Pour agréger les différents critères (étape iii), if convient d'adopter une méthode d'agrégation multicritère adaptée au contexte. Il existe trois grandes catégories de méthodes multicritères (Roy et Bouyssou, 1993) : l'utilité multiattribut, les méthodes de surclassement et les méthodes interactives. Nous avons fait le choix d'utiliser une méthode interactive où alternent des étapes de calcul et des étapes de dialogue avec le décideur. La méthode d'affectation (le tri) par l'établissement de règles que nous avons retenue est bien adaptée aux contextes d'agrégation complexe (Azibi, 2003). Elle consiste à construire une base de règles de type asi... alors.... et proches du langage naturel pour exprimer des principes d'agrégation. Cette méthode apparaît bien adaptée à notre problématique de décision: les règles permettent d'intégrer les préférences des experts des digues, l'importance relatives des critères et leurs interactions.

Dans cette partie, nous proposons une illustration de notre travail au mécanisme d'érosion interne des digues à étanchéité amont.

\section{2}

\section{Détermination des indicateurs d'état et des critères des digues}

Nous avons modélisé les mécanismes de rupture des digues sous la forme de graphes causaux. Le modèle fonctionnel repose sur trois variables: les fonctions, les phénomènes et les indicateurs. Les indicateurs renseignent sur les phénomènes auxquels ils sont 
rattachés. Ces phénomènes correspondent aux causes ou aux effets de perte de performance des composants des digues, Chaque phénomène est renseigné en agrégeant un certain nombre d'indicateurs. Enfin, l'agrégation des phénomènes renseigne sur la performance d'une fonction.

Les indicateurs d'état et les critères des digues sont donc obtenus à partir de la modélisation fonctionnelle des mécanismes:

- les indicateurs d'état correspondent aux indicateurs obtenus par l'AMDE;

- les critères correspondent aux phénomènes obtenus par l'AMDE (c'est-à-dire les causes et les effets des modes de défaillance dans l'analyse AMDE).

Enfin, les indicateurs de performance correspondent à l'évaluation des fonctions obtenues par l'analyse fonctionnelle (Fig. 11).

\section{3}

\section{Évaluation des indicateurs et des critères}

Pour renseigner les règles d'affectation des indicateurs d'état et des critères, nous avons constitué un groupe d'experts du domaine des digues, composé de deux experts seniors en ouvrages hydrauliques, un expert hydraulicien et un animateur généraliste spécialiste des SIG. Le rôle de ce groupe d'experts est de fixer l'ensemble des règles permettant de rendre la méthode opérationnelle. Les séances de travail du groupe d'experts ont été menées essentiellement sur la base d'interviews structurées, lors desquelles des arbres de défaillance issus de la représentation des scénarios ont servi de guide lors des discussions (Fig. 12).

Dans un premier temps, il est indispensable de décrire précisément les indicateurs d'état utilisés de manière à ce que leur mesure soit fiable et répétable. Un

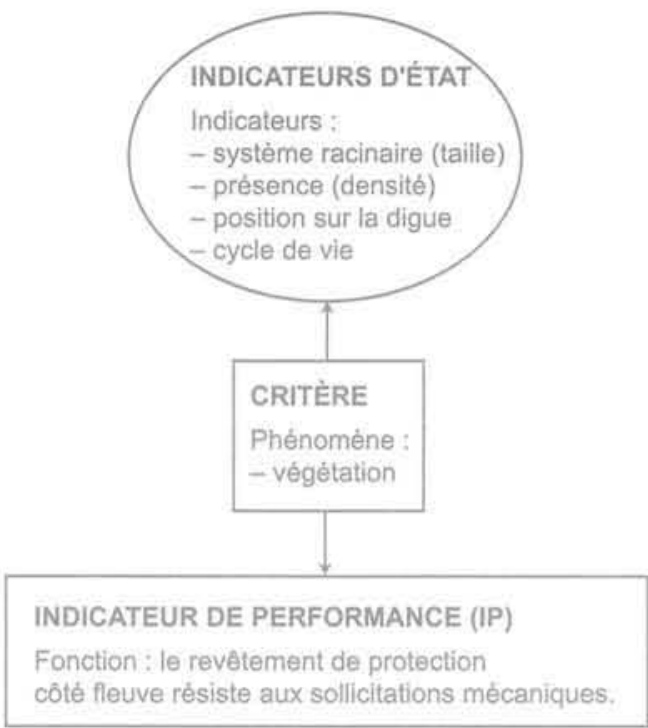

FIG.11 Indicateurs d'état, critère et indicateur de performance des digues: exemple.

Condition indicators, criteria and levee performance indicators: example.

certain nombre de champs des indicateurs d'état doivent être renseignés : nom de l'indicateur d'état, définition, mode opératoire, échelle de notation, références sur cette échelle et position géographique sur la digue.

Ensuite, nous proposons une échelle des scores applicable aux indicateurs d'état et aux critères. Nous adoptons une échelle discrète de scores variant de 0 à 10: 0 correspond à la situation idéale tandis que 10 à la situation la plus critique (Fig. 13). Cette échelle unique pour tous les indicateurs d'état et tous les critères permet d'introduire une hiérarchie dès la mesure.

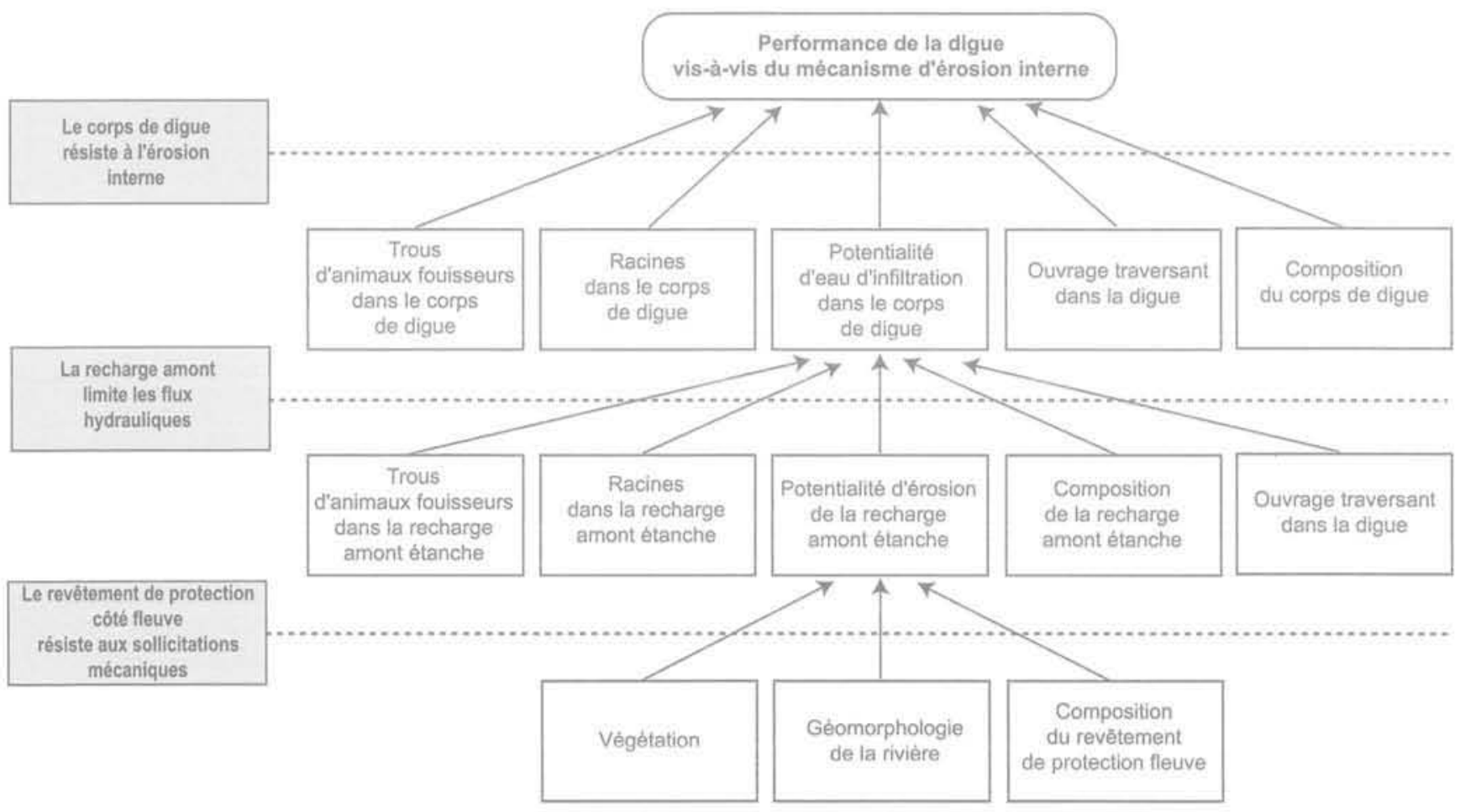

FIG.12 Arbre de défaillance pour le mécanisme d'érosion interne des digues à étanchéité amont.

Fault tree of internal erosion mechanism in levee with impervious upstream shoulder. 


\begin{tabular}{|c|c|c|}
\hline Score & Qualification & Code couleur \\
\hline 0 & Excellent & \\
\hline 1 & Bon & \\
\hline 2 & Bon & \\
\hline 3 & Passable & \\
\hline 4 & Passable & \\
\hline 5 & Médiocre & \\
\hline 6 & Médiocre & \\
\hline 7 & Mauvais & \\
\hline 8 & Mauvais & \\
\hline 9 & Mauvais & \\
\hline 10 & Inacceptable & \\
\hline
\end{tabular}

FIG. 13 Qualification des scores. Scores qualification.

\begin{tabular}{|c|c|c|c|c|c|c|c|c|c|c|c|c|}
\hline \multicolumn{13}{|c|}{ Critère a racines dans la recharge amont étanche s } \\
\hline Sous-critere & Indicateur & D & 1 & 2 & 3 & 4 & s & 6 & 7 & 8 & 9 & 10 \\
\hline \multirow[t]{3}{*}{ Grands aitbres } & contuct btancheite: & & & & & & & & & $x$ & $x$ & $x$ \\
\hline & hors dtanchasab & & & & $x$ & $x$ & $\mathrm{x}$ & $x$ & & & & \\
\hline & excolience & $x$ & & & & & & & & & & \\
\hline Sous-critère & Indicateur & 0 & 1 & 2 & 3 & 4 & 5 & 6 & 7 & 8 & 9 & 10 \\
\hline \multirow[t]{3}{*}{ Pelits arbres } & contact etancheilie & & & & & & & & $x$ & $\mathrm{x}$ & $x$ & \\
\hline & hors etanchiste & & & & $x$ & $x$ & & & & & & \\
\hline & axcellence & $x$ & & & & & & & & & & \\
\hline Sous-critëre & Indicatour & 0 & 1 & 2 & 3 & 4 & 5. & 8 & 7 & 8 & 9 & 10 \\
\hline \multirow[t]{3}{*}{ Hivisans: } & contact étancheite & & & & $x$ & $x$ & & & & & & \\
\hline & hors átanchoild & & & $x$ & $x$ & & & & & & & \\
\hline & excellenct & $x$ & & & & & & & & & & \\
\hline
\end{tabular}

FlG. 14 Grille d'analyse synthétique du critère « racines dans la recharge amont étanche $)$.

Synthetic analysis grid of criterion u roots in impervious upstream shoulder $n$.
Les experts établissent les règles applicables à l'ensemble des indicateurs d'état: les scores 0 et 10 sont fixés ainsi que les scores intermédiaires. Pour chacun des indicateurs d'état, nous fixons les règles d'évaluation faisant correspondre à chacun d'eux des scores dépendant des observations relevées sur les digues, de leurs mesures d'auscultation, des essais disponibles ou des facteurs de stabilité calculés. Ces scores vont de la condition d'excellence (situation où l'indicateur d'état prend le score 0) à la condition inacceptable (situation où l'indicateur d'état prend le score 10).

Enfin, le groupe d'experts propose une règle d'agrégation des indicateurs d'état pour obtenir une évaluation de chaque critère. Les indicateurs d'état sont combinés pour obtenir la valeur des critères à l'aide de différents opérateurs: somme, somme pondérée, maximum, table de vérité...

A titre applicatif, nous traitons l'exemple de l'évaluation du critère "racines dans la recharge amont étanchen, qui intervient dans la séquence de défaillance de la fonction d'étanchéité de la recharge amont (voir l'arbre de défaillance de la figure 12 «scénario d'érosion interne »), La règle proposée par les experts indique que la végétation examinée pour ce critère est celle située sur la digue (talus côté fleuve et crête) et à ses abords (sur une emprise de 2 mètres de part et d'autre de la recharge amont). Ce critère est évalué à partir de trois sous-critères: «grands arbres », "petits arbres») et "buissons », chacun de ces sous-critères étant eux-mêmes évalué à partir de deux indicateurs d'état: "végétation en contact avec le dispositif d'étanchéité » et "végétation hors du dispositif d'étanchéité nn (Fig. 14).

La condition d'excellence (score 0) est obtenue si aucun arbre ou buisson ne se situe sur la digue ou à proximité (sur une emprise de 2 mètres de la digue). La condition inacceptable (score 10) est atteinte dès qu'un seul grand arbre mort est observé au contact de la recharge amont étanche ou à proximité (à moins de 2 mètres de la recharge). L'agrégation des trois sous-critères pour l'évaluation du critère «racines dans la recharge amont étanche » est obtenue en adoptant le score le plus défavorable (opérateur Maximum). Le détail des règles est résumé dans une grille d'analyse synthétique (Fig. 14),
Pour l'ensemble des critères intervenants dans le scénario d'érosion interne (13 critères au total), les experts ont fixé de façon analogue les règles : les conditions d'excellence et inacceptables des indicateurs d'état, leurs règles d'affectation des scores et leurs règles d'agrégation pour l'évaluation des critères.

\section{4}

\section{Évaluation de la performance des digues}

A ce stade, nous disposons de règles pour l'évaluation des critères intervenants dans les scénarios de rupture des digues. Il reste à proposer des règles d'agrégation des critères pour l'évaluation de la performance de la digue vis-à-vis d'un mécanisme donné.

Les critères doivent être agrégés à chaque séquence fonctionnelle du scénario (voir arbre de défaillance, Fig. 12). Les résultats de l'agrégation de chacune des séquences de défaillance sont ensuite reportés à la séquence suivante du scénario et ce, jusqu'à l'évaluation de la performance de la fonction principale (c'està-dire la performance de la digue vis-à-vis du mécanisme étudié, figurant au sommet de l'arbre de défaillance, Fig. 12).

Nous présentons cette démarche d'agrégation des critères sur l'exemple du mécanisme d'érosion interne des digues à étanchéité amont (Fig. 12). La première séquence du scénario, correspondant à la performance de la fonction de résistance du revêtement amont (fonction «le revêtement de protection côté fleuve résiste aux sollicitations mécaniques $)$ ), fait intervenir trois critères: "végétation , " géomorphologie de la rivière » et "composition du revêtement côté fleuve ». Ces trois critères sont agrégés par l'opérateur Maximum pour l'obtention du score du critère "potentialité d'érosion de la recharge arnont $n$, apparaissant dans la deuxième séquence de défaillance. De façon analogue, cette même règle est utilisée pour évaluer le critère " potentialité d'eau d'infiltration dans le corps de digue » de la troisième séquence de défaillance.

Enfin, pour évaluer la performance de la digue visà-vis du mécanisme d'érosion interne, deux séries de critères sont agrégées: 
- le critère "cpotentialité d'eau d'infiltration dans le corps de digues $»$ obtenu ci-dessus;

- l'agrégation par l'opérateur Maximum des quatre critères « trous d'animaux fouisseurs dans le corps de digues», "racines dans le corps de digue», "ouvrage traversant dans la digue $)$ et «composition du corps de digue ).

Cette agrégation est réalisée à l'aide d'une table de vérité (Fig. 15), établie par le groupe d'experts.

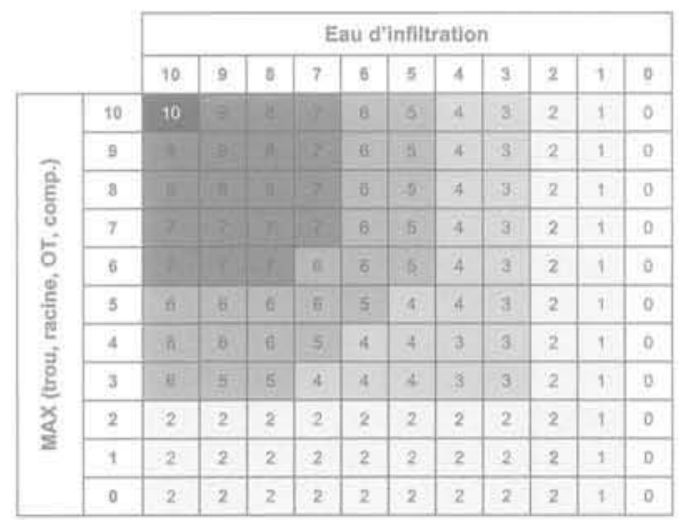

FIG. 15 Table de vérité experte pour évaluer la performance d'une digue vis-à-vis de I'érosion interne.

Expert's truth table to assess the levee performance related to internal erosion mechanism

\section{5}

\section{Synthèse de l'évaluation de la performance des digues}

Après avoir énoncé les principes généraux de construction des indicateurs de performance, nous avons bâti un système d'indicateurs d'état et de critères pour évaluer la performance des digues. Les indicateurs d'état utilisés sont déterminés à partir de l'AMDE lors de la construction des scénarios de rupture. Ils sont évalués par les experts du domaine des digues pour chaque scénario de rupture avec une échelle commune de notation allant de 0 à 10. A partir des scores de chaque indicateur d'état, l'évaluation des critères est obtenue par l'application de l'opérateur Maximum.

Nous avons répondu à notre problème multicritère par la méthode d'affectation à base de règles. Sa mise en ceuvre sur les digues permet d'évaluer, à partir des différents critères, la performance des digues pour chaque mécanisme de rupture.

\section{Conclusion}

Notre recherche a consisté à produire des méthodes pour évaluer la performance des digues de protection contre les inondations, l'objectif étant de fournir aux gestionnaires des outils d'aide à la décision pour la planification des programmes d'inspection, de maintenance et de réparation.

Dans une première étape, nous avons mis en œuvre des méthodes de la Sûreté de Fonctionnement: l'analyse fonctionnelle, l'analyse des modes de défaillance et de leurs effets et une représentation par des arbres de défaillance. Ces méthodes ont permis de modéliser la structure des digues, de connaître les fonctions accomplies par les digues et leurs composants, et de définir leurs modes de défaillance, leurs causes et leurs effets. A partir du recueil et de la formalisation de la connaissance experte, nous avons modélisé les principaux mécanismes de rupture des digues sous forme de scénarios. Pour chacun des mécanismes, nous déterminons les indicateurs d'état et les critères qui interviennent dans l'évaluation de la performance. En terme de résultats, nous avons modélisé les mécanismes de rupture des digues (6 scénarios pour les digues à étanchéité amont) et nous avons déterminé, pour chaque mécanisme, les critères (quelque 150 critères pour l'ensemble des mécanismes de rupture).

L'étape suivante a consisté à agréger les indicateurs d'état et les critères pour évaluer la performance des digues. La méthode utilisée est une méthode multicritère d'affectation à base de règles, alternant des étapes de discussions avec un groupe d'experts et des étapes de calcul. En terme de résultats, cette démarche a permis d'établir les règles d'évaluation et d'agrégation des critères (quelque 120 règles pour le mécanisme de rupture par érosion interne des digues à étanchéité amont couvrant 13 critères), permettant in fine l'évaluation de la performance des digues (6 mécanismes pour les digues à étanchéité amont).

Pour compléter ces résultats, des travaux complémentaires vont être réalisés à court terme suivant deux directions:

- un axe de recherche visant à poursuivre et achever l'évaluation de la performance des digues pour l'ensemble des mécanismes de rupture et pour les trois modèles structurels de diques. En outre, la vérification détaillée de la capacité des méthodes multicritères d'évaluation de la performance des digues à représenter les situations réelles devra être poursuivie;

- un axe de développement destiné à produire un véritable outil opérationnel. Sur la base d'un SIG existant dans lequel nous avons intégré les règles multicritères d'évaluation de la performance des digues (Maurel et al., 2004), il s'agira de produire un outil d'aide à la décision destiné aux gestionnaires de digues pour l'optimisation des opérations de maintenance et de réhabilitation des digues. 
Azibi R. - Construction de critères en aide à la décision : aspects méthodologiques, techniques et pratiques. Thèse de doctorat. université Paris Dauphine, UFR Sciences des organisations, 2003, 160 p

CFGB - Erosion interne: typologie, détection et réparation. Barrages et Réservoirs $n^{\circ} 6$, Le Bourget-du-Lac, CFGB. EDF, 1997, 126 p.

Diab Y, - La gestion du patrimoine enterré approche multicritère d'analyse des risques. INFRA, Montréal, Québec, 2002, $7 \mathrm{p}$

Fauchard C. Mériaux P. - Méthodes géophysiques et géotechniques pour le diagnostic des digues de protection contre les crues. Guide pour la mise en gervre et l'interprétation. Cemagref Éditions, 2004, 124 p.

Le Gauffre P. Laffréchine $\mathrm{K}$., Haidar $\mathrm{H}$. Baur R. Poinard D, Schiatti M. - A multicriteria decision support tool for annual rehabilitation programmes of water networks. Principles and experi- ments, DMUCE 4, Porto, 28-30 pctobre 2004, $10 \mathrm{p}$.

Maurel P. Serre D.. Tourment R. Towards a generic GIS for dike management in flood plain areas : from conceptual design to real applications. AGILF 2004 conference on Geographic Information Science, April 29th-May 1st, Heraklion, Greece, 2004, 14 p.

Mériaux P., Royet P. Folton C. - Surveillance, entretien et diagnostic des digues de protection contre les inondations. Cemagref Editions, 2001, 191 p.

Mèriaux. P. Tourment R., Wolff M. - Le pa-trimoine de digues de protection contre les inondations en France. Sécurité des digues fluviales et de navigation. CFGB-MEDD, Orléans, France, 2004, p. 9-20.

Peyras L - Diaqmostic et analyse de risques liés au vieillissement des barrages, déve loppement de méthodes d'aide à l'exper tise. Thèse de doctorat, université BlaisePascal, Clermont 2, 2003, 199 p.
Pilarczyk K.W. - Dikes and revetments. Design, maintenance and safety assess. ment. A.A. Balkema, Rotterdam, Netherlands, $1998,562 \mathrm{p}$.

Roy B. Bouyssou D. - Aide multicritère la décision: méthodes et cas. Éditions Economica, Paris, 1993, 695 p.

Serre D, - Evaluation de la performance des digues de protection contre les inondafions. Modélisation de critères de décision dans un Système d'Information Géographique. Thèse de doctorat, université de Marne-la-Vallée, 2005, 240 p.

Zwingelstein G. - La maintenance basée sur la fiabilité. Guide pratique d'application de la RCM. Hermès Éditions, 1996. $666 \mathrm{p}$.

Villemeur A. - Süreté de fonctionnement des systèmes industriels. Fiabilité. Facteurs humains-Informatisation, "Collection de la Direction des Études et Recherches d'Électricité de France $n$. Editions Eyrolles, 1988, p. 121-141. 